\title{
Influencia de la carga inicial de glucosa sobre los pa- cientes en diálisis peritoneal
}

\section{Francisco Cirera Segura - Jesús Lucas Martín Espejo}

Unidad Uro-Nefrológica. H.H.U.U. Virgen del Rocío. Sevilla

\section{Resumen}

Los objetivos del estudio fueron determinar si diferentes cargas de glucosa en el inicio de la diálisis peritoneal pueden ser negativas para la supervivencia de la técnica o de los pacientes y conocer qué factores les afectan y cómo puede actuar Enfermería al respecto.

Se realizó un estudio retrospectivo desde 2004 a 2008. La muestra la constituyeron 63 pacientes, clasificados en 3 grupos según la concentración de glucosa. Se recogieron datos epidemiológicos, analíticos, transporte y aclaramiento peritoneal, comorbilidad y pauta de diálisis.

Los resultados demuestran que los tres grupos se diferenciaban en el peso total de glucosa $(p=0,001)$, en su concentración media $(p<0,001)$, en la albúmina sérica $(p<0,05)$, y el cociente dializado-plasma de creatinina $(p=0,036)$.

Tuvieron lugar 15 fallecimientos, no encontrando diferencias significativas para la supervivencia de los pacientes entre los grupos $(p=0,163)$, aunque la supervivencia del primer año fue del 95,2\%, $94,1 \%$ y $78,4 \%$. Los factores que influyeron en la supervivencia fueron la edad, la enfermedad cardiovascular, el Kt/V total, la diuresis y la albúmina. El fallo de la técnica tuvo lugar en 8 pacientes y no hubo diferencias entre los grupos $(p=0,769)$, ni factores independientes que influyeran en la supervivencia de la técnica.

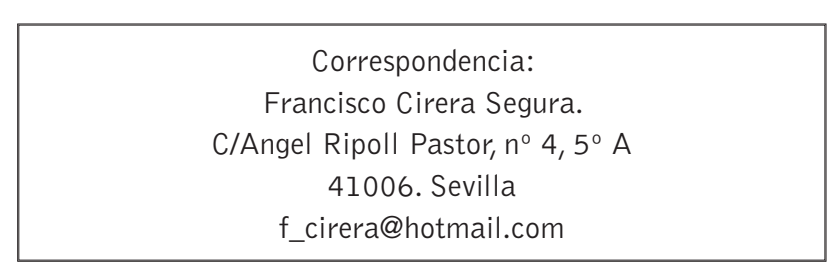

Como conclusión, no hemos podido demostrar que una mayor carga de glucosa inicial, afecte a la supervivencia de los pacientes o a la técnica.

PALABRAS CLAVE:

- DIÁLISIS PERITONEAL

- GLUCOSA

- AGENTE OSMÓTICO

- SUPERVIVENCIA

- ENFERMERÍA

\section{Influence of the initial glucose load on pa- tients undergoing peritoneal dialysis}

\section{Abstract}

The aims of the study were to determine whether different glucose loads at the start of peritoneal dialysis could be negative for survival of the technique or of the patients, and to find out what factors affect them and how nursing staff can act in this regard.

A retrospective study was carried out from 2004 to 2008. The sample comprised 63 patients, classified in 3 groups according to the glucose concentration. Epidemiological, analytical, peritoneal transport and rinsing, comorbility and dialysis dose data were compiled.

The results show that the three groups were differentiated by the total glucose weight $(p=0.001)$, average concentration $(p<0.001)$, serum albumin $(p<0.05)$, and the dialysate/creatinine plasma quotient $(p=0.036)$.

There were 15 deaths, with no significant differences found for the survival of the patients between 
the groups ( $p=0.163)$, although first-year survival was $95.2 \%, 94.1 \%$ and $78.4 \%$. The factors that influenced survival were age, cardiovascular disea$\mathrm{se}$, total $\mathrm{Kt} / \mathrm{V}$, diuresis and albumin. Failure of the technique occurred in 8 patients and there were no differences between the groups $(p=0.769)$, nor any independent factors affecting the survival of the technique.

In conclusion, we have been unable to prove that a higher initial glucose load affects the survival of the patients or of the technique.

\section{KEY WORDS: \\ - PERITONEAL DIALYSIS \\ - GLUCOSE \\ OSMOTIC AGENT \\ - SURVIVAL \\ - NURSING}

\section{Introducción}

El empleo de soluciones de diálisis peritoneal (DP) basadas en distintas concentraciones de glucosa, es y ha sido durante más de dos décadas, el método habitual para obtener la ultrafiltración a través del peritoneo. Aunque hoy en día existen otros agentes osmóticos como los aminoácidos y los polímeros de glucosa, aún sigue siendo el más utilizado. La glucosa se ha mostrado un agente osmótico efectivo, seguro, metabolizable, barato y además proporciona un aporte energético diario ${ }^{1}$.Como desventaja, el peritoneo puede permitir una rápida absorción de la glucosa del dializado con pérdida progresiva de su gradiente osmótico, de tal forma que en permanencias largas, se puede reabsorber parte del líquido peritoneal y no obtener una ultrafiltración adecuada. Esta absorción excesiva de glucosa puede provocar hiperglucemia, dislipemia y obesidad, con las complicaciones que ello conlleva: empeorar el síndrome metabólico y aumentar el riesgo de enfermedad cardiovascular 2-3-4-5-6. Recientemente Szeto et $\mathrm{al}^{7}$, publicaron que la aparición en el primer mes de hiperglucemia en los pacientes que inician DP y no eran diabéticos previamente, predecía peores resultados.
El peritoneo es una membrana biológica que en DP se expone reiteradamente al contacto con líquidos no biocompatibles que contienen glucosa, lactato, y una alta osmolaridad, por lo que aumentan los productos de degradación y glicosilación no enzimática de la glucosa ${ }^{8-9-10}$, dañándolo, y por tanto, afectando a la supervivencia del paciente y de la técnica ${ }^{1-2}$.

Estudios previos sugieren que mayores exposiciones del peritoneo a la glucosa pueden causar daños morfológicos y funcionales ${ }^{2}$, y repercutir sobre el transporte peritoneal ${ }^{9}$, incluso se ha relacionado la peritonitis esclerosante al uso de cambios con glucosa hipertónica ${ }^{11-12}$. Aún así, su utilización es necesaria para obtener una ultrafiltración mínima adecuada ${ }^{13}$.

Por estos motivos, los objetivos de este estudio son determinar si las diferentes cargas de glucosa utilizadas en el periodo inicial de la terapia en DP pueden tener un impacto negativo tanto para la supervivencia de la técnica como en la de los pacientes en DP, y tratar de conocer qué factores les afecta y cómo puede actuar Enfermería al respecto.

\section{Material y Método}

Se ha realizado un estudio retrospectivo de los pacientes incidentes en nuestra unidad, desde enero de 2004 a diciembre de 2008. Los criterios de exclusión fueron: no tener un test de equilibrio peritoneal (TEP) en los 3 primeros meses o tener una supervivencia en la técnica menor de 3 meses al inicio del estudio. De los 73 pacientes que iniciaron DP durante el estudio, se excluyeron 9 por no disponer del TEP y 1 por tener una supervivencia en la técnica menor de 3 meses. Por tanto, la muestra la constituyeron 63 pacientes.

Al inicio del estudio se recogieron los siguientes datos epidemiológicos: edad, sexo, altura, peso, índice de masa corporal (IMC) y etiología de la ERC ${ }^{14}$, así como los datos analíticos (urea, creatinina, y albúmina), el transporte y el aclaramiento peritoneal, en los 3 primeros meses desde el inicio en DP. El trasporte peritoneal se evaluó mediante el TEP, como describe Twardowski ${ }^{15}$. El aclaramiento de urea (Kt/V) se calculó a través del programa P-Adequest ${ }^{\circledR}$. La ade- 
cuación de la diálisis (Kt/V Total) se obtuvo sumando el Kt/V renal y peritoneal semanal. La hipoalbuminemia se definió como la albúmina sérica menor de $3,5 \mathrm{~g} / \mathrm{dl}$.

Para valorar la comorbilidad se utilizó la escala de Charlson distinguiendo entre: diabetes pre-existente, enfermedad cardiovascular, enfermedad hepática 0 pulmonar. Se definió con sobrepeso a aquellos pacientes con un IMC superior a $25 \mathrm{Kg} / \mathrm{m}^{2} .{ }^{16}$ Se recogió la pauta de diálisis prescrita en los 6 primeros meses en DP. El producto del volumen y la concentración de glucosa de cada intercambio fue calculado como describen Davies et al ${ }^{9}$. La media de la concentración de glucosa se calculó dividendo el peso total de glucosa por el total del volumen de dializante prescrito en los 3 primeros meses. Los pacientes podían usar líquidos de DP que no utilizaran glucosa, cuyo volumen se tuvo en cuenta en el cálculo de la concentración media de glucosa. No se discriminó para el estudio el tampón utilizado en los líquidos de DP.

Los sujetos se clasificaron en 3 grupos según la concentración media de glucosa:

- Grupo 1. Glucosa Baja (hasta 1,43\%)

- Grupo 2. Glucosa Media (entre 1,44\% - 1,77\%)

- Grupo 3. Glucosa Alta (mayor de 1,77\%)

Los resultados se expresaron con la media y la desviación estándar o en frecuencias y porcentajes según fuese apropiado. Cuando las variables no cumplieron la distribución normal, comprobada mediante la prueba de Kolmogorov, se utilizó la mediana y el rango. Para la comparación de medias se utilizó la prueba de Kruskal-Wallis o ANOVA de un factor, la $X^{2}$ para las variables cualitativas, y en el estudio de correlaciones el estadístico de Pearson o la Rho de Spearman en función de las variables. El estudio de supervivencia se realizó mediante curvas de KaplanMeier y se compararon con el test de Log-Rank (Mantel-Cox). Para la supervivencia de los pacientes, el evento final fue el fallecimiento y como perdidos se consideraron el cambio de tratamiento y el trasplante renal. Para la supervivencia de la técnica, el evento final fue el paso a hemodiálisis y se consideraron perdidos el trasplante renal y el fallecimiento. Para analizar los factores de riesgo en la supervivencia del paciente y la técnica se utilizó la regresión de Cox, en primer lugar univariante, introduciendo las variables con $p<0,2$ en el análisis multivariante. Los valores de $p>0,05$ fueron considerados significativos. Se utilizó el paquete estadístico SPSS 16.0.

\section{Resultados}

La muestra la compusieron un total 63 pacientes, 35 hombres y 28 mujeres, con una edad media de 61,27 $\pm 15,27$ años al inicio de la DP. El 63,49\% $(n=40)$ estaban en diálisis peritoneal continua ambulatoria (DPCA) y $36,51 \% \quad(n=23)$ en diálisis peritoneal automática (DPA). El tiempo medio en DP fue de $17,76 \pm 10,26(4-51)$ meses. La nefropatía causal de la ERC se muestra en la figura 1.

El 34,9\% ( $n=22)$ de los pacientes tenían una edad superior a 70 años, el 69,8\% ( $n=44)$ tenía sobrepeso y el $34,9 \%(n=22)$ hipoalbuminemia. Respecto al tipo de trasporte peritoneal al inicio del estudio, el $1,6 \%$ de los pacientes se clasificó como bajo trasportador, un $17,5 \%$ medio-bajo, un $52,4 \%$ como medioalto y un $14,5 \%$ como alto. Las características de los pacientes agrupadas según la carga de glucosa utilizada así como la prevalencia de las enfermedades comorbiles se muestran en la tabla 1.

Los 3 grupos estuvieron formados por 21 pacientes y se encontraron diferencias estadísticamente significativas en el peso total de glucosa $(p=0,001)$, la concentración media de glucosa $(p<0,001)$, la albúmina sérica $(p<0,05)$, y el cociente dializado-plasma $(D / P)$ de creatinina $(p=0,036)$.

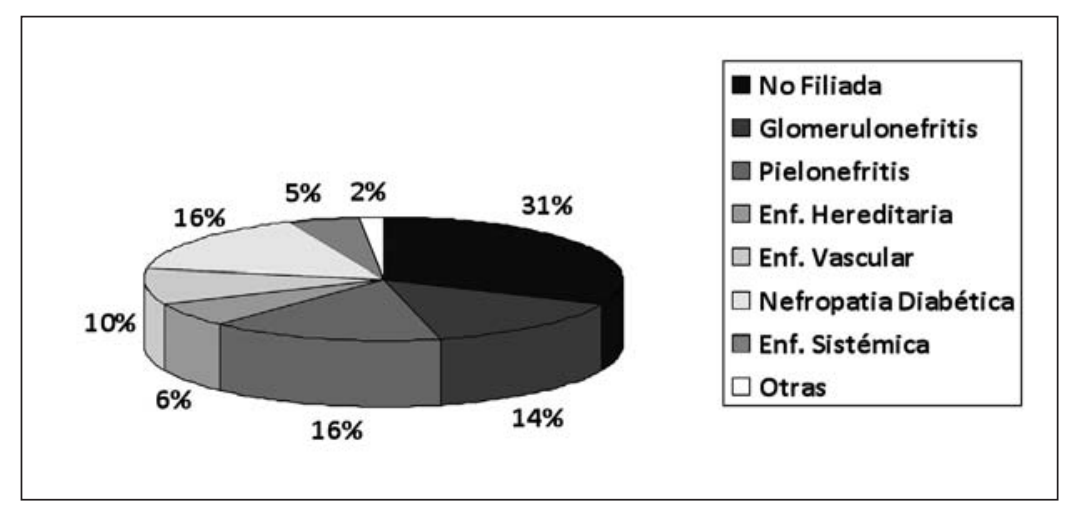

Figura 1. Patología Base de la ERC 


\begin{tabular}{|c|c|c|c|c|c|}
\hline & & & Glucosa Baja & Glucosa Media & Glucosa Alta \\
\hline \multicolumn{3}{|l|}{ Pacientes } & $n=21$ & $n=21$ & $n=21$ \\
\hline \multicolumn{3}{|c|}{ Hombres: Mujeres } & $12: 9$ & $12: 9$ & $11: 10$ \\
\hline \multicolumn{3}{|c|}{ Edad (años) } & $60,10 \pm 12,52$ & $58,67 \pm 18,48$ & $65,95 \pm 13,93$ \\
\hline \multicolumn{3}{|c|}{ Tiempo en DP } & $17,86 \pm 11,57$ & $21 \pm 10,22$ & $14,43 \pm 8,09$ \\
\hline \multicolumn{3}{|c|}{ Peso Total de Glucosa (g) } & $18702,93 \pm 8859,06^{\mathrm{bc}}$ & $23527,3 \pm 6306,29^{b}$ & $30479,39 \pm 9850,25$ \\
\hline \multicolumn{3}{|c|}{ Concentración Media de Glucosa } & $1,12 \%$ bc & $1,60 \%$ & $2,01 \%$ \\
\hline \multicolumn{3}{|c|}{ Altura $(\mathrm{cm})$} & $163,24 \pm 10,16$ & $162,95 \pm 10,38$ & $158,14 \pm 12,61$ \\
\hline \multicolumn{3}{|c|}{ Peso $(\mathrm{Kg})$} & $71,88 \pm 15,47$ & $70,71 \pm 15,12$ & $70,69 \pm 10,35$ \\
\hline \multirow{4}{*}{\multicolumn{2}{|c|}{ Comorbilidad }} & DM Previa & $6(28,6 \%)$ & $6(28,6 \%)$ & $4(19 \%)$ \\
\hline & & Cardiovascular & $12(57,1 \%)$ & $12(57,1 \%)$ & $14(66,7 \%)$ \\
\hline & & Hepática & $2(9,5 \%)$ & $1(4,8 \%)$ & $2(9,5 \%)$ \\
\hline & & Pulmonar & $5(23,8 \%)$ & $4(19 \%)$ & $3(14,3 \%)$ \\
\hline \multicolumn{3}{|c|}{ Sobrepeso (IMC>25kg/m²) } & $13(61,9 \%)$ & $13(61,9 \%)$ & $18(85,7 \%)$ \\
\hline \multicolumn{3}{|c|}{ D/P Creatinina } & $0,77 \pm 0,12^{c}$ & $0,69 \pm 0,08^{b}$ & $0,76 \pm 0,1$ \\
\hline \multicolumn{3}{|l|}{ Kt/V Total } & $2,61 \pm 0,92$ & $2,4 \pm 0,58$ & $2,43 \pm 0,9$ \\
\hline \multicolumn{3}{|c|}{ Orina 24 Horas $(\mathrm{mL})$} & $1032,95 \pm 595,92$ & $873,81 \pm 555,56$ & $765 \pm 712,13$ \\
\hline \multirow{3}{*}{ Analítica } & & $(\mathrm{mg} / \mathrm{dL})$ & $172,05 \pm 68,63$ & $174,63 \pm 70,50$ & $154,28 \pm 50,17$ \\
\hline & & tinina ( $\mathrm{mg} / \mathrm{dL})$ & $7,94 \pm 1,84$ & $7,95 \pm 1,87$ & $7,62 \pm 1,39$ \\
\hline & & $\operatorname{nina}(g / d L)$ & $3,7 \pm 0,3^{b}$ & $3,4 \pm 0,4$ & $3,3 \pm 0,6$ \\
\hline \multirow{3}{*}{$\begin{array}{l}\text { Causa de } \\
\text { Salida de } \\
\text { DP }\end{array}$} & & lante Renal & $3(14,3 \%)$ & $3(14,3 \%)$ & $2(9,5 \%)$ \\
\hline & & a Hemodiálisis & $4(19 \%)$ & $2(9,5 \%)$ & $2(9,5 \%)$ \\
\hline & & & $3(14,3 \%)$ & $5(23,8 \%)$ & $7(33,3 \%)$ \\
\hline
\end{tabular}

Tabla 1. Datos Demográficos y Clínicos, según la Carga de Glucosa.

Los pacientes de mayor edad tenían un mayor peso e IMC $(p=0,008 r=0,331)$. Mayores niveles de albúmina se correlacionaron: con una mayor diuresis $(p=0,013 r=0,312)$, un $D / P$ creatinina menor $(p=0,006 r=-0,352)$, una menor edad de los pacientes $(p=0,44 r=-0,254)$ y una menor concentración de glucosa media ( $p=0,022 r=-0,289)$. Una mayor cantidad total de glucosa se correlacionó: con mayores Kt/V peritoneales $(p=0,01$ $r=0,327)$ y con la DPA como modalidad de DP $(p<0,001 r=0,515)$.

Durante el periodo de seguimiento, tuvieron lugar 15 fallecimientos (23,8\%). En el 73,35\% de los pacientes la causa fue la enfermedad cardiovascular, en un $20 \%$ infecciosa, y en el $6,6 \%$ otras causas. No se objetivó una diferencia sig- nificativa para la supervivencia de los pacientes entre los tres grupos de glucosa $(p=0,163)$, aunque en el primer año la supervivencia fue del 95,2\%, 94,1\% y $78,4 \%$ para cada grupo respectivamente (figura 2 ).

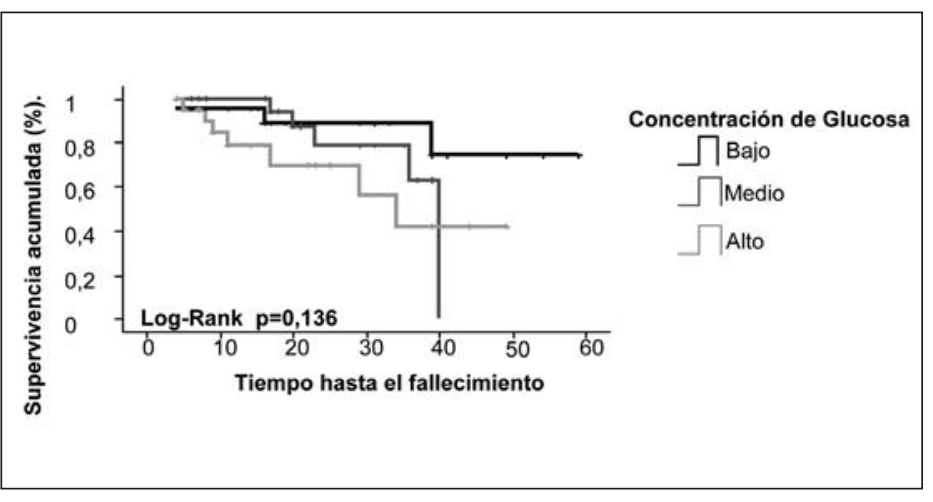

Figura 2. Kaplan-Meyer para la Supervivencia de los Pacientes. 
En el análisis de los factores independientes que influyeron en la supervivencia de los pacientes, se encontraron como tales: la edad, la enfermedad cardiovascular el $\mathrm{Kt} / \mathrm{V}$ total, la diuresis y la albúmina sérica, aunque en el modelo multivariante sólo la diuresis de 24 horas $(p<0,001)$, la albúmina sérica $(p=0,001)$ y la existencia previa de enfermedad hepática $(p=0,027)$ fueron significativas para la supervivencia de los pacientes (tabla 2 ).

El fallo de la técnica con trasferencia a hemodiálisis, tuvo lugar en 8 pacientes $(12,69 \%)$ y no se objetivaron diferencias significativas entre los grupos, $p=0,769$ (figura 3 ). No se encontraron factores independientes que influyeran en la supervivencia de la técnica.



Figura 3. Kaplan-Meyer para la Supervivencia de la Técnica.

\begin{tabular}{|c|c|c|c|c|}
\hline & \multicolumn{2}{|c|}{ Análisis Univariante } & \multicolumn{2}{|c|}{ Análisis Multivariante } \\
\hline & $\mathrm{RR}$ & $95 \%$ IC & $\mathrm{RR}$ & $95 \%$ IC \\
\hline Edad (comparado con $>70$ ) & 3,566 & $1,233-10,311^{f}$ & - & - \\
\hline Hombres vs Mujeres & 0,86 & $0,306-2,421$ & - & - \\
\hline Sobrepeso (IMC>25) & 2,021 & $0,569-7,176$ & - & - \\
\hline Diabetes M. Previa & 0,901 & $0,285-2,856$ & - & - \\
\hline Enf. Cardiovascular & 8,696 & $1,141-66,303^{f}$ & - & - \\
\hline Enf. Pulmonar & 1,183 & $0,329-4,252$ & - & - \\
\hline Enf. Hepática & 3,297 & $0,707-15,381$ & 10,069 & $1,531-66,243^{f}$ \\
\hline D/P Creatinina & 3,176 & $0,042-242,95$ & - & - \\
\hline Kt/V Total & 0,392 & $0,173-0,887^{f}$ & - & - \\
\hline Diuresis 24 horas & 0,997 & $0,996-0,999^{d}$ & 0,998 & $0,997-0,999^{e}$ \\
\hline Albúmina Sérica & 0,133 & $0,048-0,366^{d}$ & 0,122 & $0,032-0,470^{e}$ \\
\hline \multicolumn{5}{|l|}{ Carga de glucosa } \\
\hline Baja $^{a}$ & 2,502 & $0,699-8,958$ & - & - \\
\hline Media ${ }^{b}$ & 1,096 & $0,37-3,244$ & - & - \\
\hline Alta $^{c}$ & 0,41 & $0,148-1,137$ & - & - \\
\hline
\end{tabular}

Tabla 2. Factores Independientes para la Supervivencia del Paciente

\section{Discusión}

En este estudio hemos intentado analizar la repercusión de la glucosa sobre la supervivencia de los pacientes y de la propia técnica de DP y no hemos encontrado diferencias significativas en ninguna de las dos. Sí hemos observado un mayor número de fallecimientos en el grupo de glucosa alta así como una mayor mortalidad, sobretodo en el primer año de tratamiento. Wu $\mathrm{HY}^{1}$ objetivó que una mayor carga de glucosa inicial predecía una peor supervivencia de la técnica, y casi de los pacientes, quizás la diferencia en los hallazgos se deba al tamaño de su muestra, claramente mayor. 
En la nuestra, la causa más frecuente de muerte fue la cardiovascular, que es la principal causa de mortalidad de los pacientes urémicos en diálisis, y a la que contribuyen la DM, la hiperlipemia y la obesidad, que son reconocidos factores de riesgo para el desarrollo de aterosclerosis y sus complicaciones ${ }^{17}$. Cabe destacar el alto porcentaje de pacientes con sobrepeso $(69,8 \%)$, y la influencia que la glucosa puede tener a largo plazo sobre los mismos. Además, un 34,9\% de los pacientes es mayor de 70 años y hemos comprobado que la edad está directamente relacionada con un mayor IMC y peso. Sólo en el $20 \%$ de los casos la causa de fue infecciosa.

Acerca de la supervivencia de los pacientes, y a través del análisis multivariante encontramos que la existencia de enfermedad hepática previa, la función renal residual y la albúmina sérica fueron predictores independientes para la misma.

Analizando los resultados, podemos afirmar que la hipoalbuminemia es un factor determinante para la supervivencia del los pacientes que ya se ha descrito con anterioridad, y suele asociarse con la sobrecarga de líquidos y una peor función renal residual. La albúmina sérica se correlaciona con el tipo de peritoneo, de forma que los altos trasportadores tienen menores niveles de albúmina sérica. Al inicio de la DP, existe una elevada proporción de altos trasportadores, que tras un periodo de tiempo en la técnica se estabilizan. Este hecho unido a la DM preexistente puede contribuir a la hipoalbuminemia y a una mayor mortalidad de los pacientes.

La conservación de la función renal residual, influye en la calidad de vida de los pacientes, y en DP se ha demostrado que su mantenimiento tiene una repercusión directa sobre la supervivencia de los pacientes. Un tema controvertido es la importancia de la modalidad de DP sobre su conservación, siendo la DPA la que parece implicar una mayor disminución de la función renal residual, quizás en relación con una mayor cantidad de glucosa utilizada. Además un alto cociente $\mathrm{D} / \mathrm{P}$ de creatinina se asocia con una reducción más rápida de la función renal residual. Respecto a la supervivencia de la técnica, no encontramos diferencias entre los 3 grupos según la carga de glucosa en los que se dividió la muestra, ni factores predictores para la misma.
Por estos motivos, la actividad de Enfermería debería ir encaminada a educar a los pacientes acerca de los aspectos que podrían mejorar la supervivencia de los pacientes (la sobrecarga hídrica, la hipoalbuminemia, y la conservación de la función renal residual) a través de Diagnósticos de Enfermería tales como Conocimientos deficientes relacionados con manejo inadecuado de la ingesta de líquidos o con un desequilibrio nutricional por exceso o defecto. La educación a través de estos diagnósticos ayudaría a los pacientes a manejar mejor el balance hídrico evitando o disminuyendo el uso de soluciones de glucosa hipertónica, manteniendo la función renal residual, y asegurando una ingesta adecuada de proteínas.

Como conclusión y respondiendo al objetivo del estudio, no hemos podido demostrar que una mayor carga de glucosa en los primeros meses de tratamiento en DP, afecte a la supervivencia de los pacientes 0 de la técnica, aunque sí lo hemos encontrado en otros estudios, donde un mayor aporte de glucosa en este periodo, afectó de forma negativa la supervivencia de la técnica, por lo que creemos necesarios que se amplíen estos estudios con un mayor número de pacientes.

Como Enfermeros y a través de los Diagnósticos de Enfermería, podemos educar a los pacientes y vigilar la aparición de los factores de riesgo como la hipoalbuminemia o la conservación de la función renal residual para mejorar la supervivencia de nuestros pacientes.

Por otro lado, deberíamos investigar sobre el uso de otros agentes osmóticos, como la icodextrina o los aminoácidos que parecen dañar menos el peritoneo y ayudan a mantener la función renal residual, proporcionando ultrafiltraciones adecuadas.

\section{Agradecimientos}

A la Dra. Ana Ruiz, responsable del programa de Diálisis Peritoneal de los Hospitales Universitarios Virgen del Rocío, por su colaboración y asesoramiento a la hora de realizar este trabajo. 


\section{Bibliografía}

1. Wu HY, Hung KY, Huang JW, et al. Initial glucose load predicts technique survival in patients on chronic peritoneal dialysis. Am. J. Nephrol. 2008; 28(5):765-71.

2. Sitter T, Sauter M. Impact of glucose in peritoneal dialysis: saint or sinner? Perit Dial Int. 2005; 25(5):415-25.

3. Martikainen T., Teppo A.-M., Gronhagen-Riska C. and Ekstrand A. Benefit of glucose-free dialysis solutions on glucose and lipid metabolism in peritoneal dialysis patients. Blood Purif. 2005; 23(4): 303-310.

4. Jiang N, Qian J, Lin A, lindholm B, Axelsson J, Yao Q. Initiation of glucose-based peritonel dialysis is associated with increased prevalence of metabolic syndrome in non-diabetic patients with end-stage renal disease. Blood Purif. 2008; 26(5):423-8.

5. Jaki M, Stipani S, Mihaljevi D, et al. The impact of glucose absorbed from dialysis solution on body weight gain in peritoneal dialysis treated patients. Lijec Vjesn. 2005; 127(5-6):116-120.

6. Coronel F. Contribución de las nuevas soluciones peritoneales a la evolución del paciente en diálisis peritoneal. Rev Soc. Esp Enferm Nefrol. $2^{\circ}$ Trimestre. 1999; (6):27-30.

7. Szeto CC, Chow KM, Kwan BC, et al. New-onset hyperglycemia in nondiabetic Chinese patients started on peritoneal dialysis. Am J Kidney Dis. 2007; 49:524-532.

8. Do JY, Kim YL, Park JW, Cho KH, Kim TW, Yoon KW, Kim CD, Park SH, Han JH, Song IH. The effect of low glucose degradation product dialysis solution on epithelial-to-mesenchymal transition in continuous ambulatory peritoneal dialysis patients. Perit Dial Int. 2005 Feb; 25 Suppl 3:S22-5.
9. Davies SJ, Phillips L, Naish PF, Russell GI. Peritoneal glucose exposure and changes in membrane solute transport with time on peritoneal dialysis. $J$ Am Soc Nephrol. 2001 May; 12(5):1046-51.

10. Witowski J, Jorres A, Koryalska K, Ksiazek K, et al. Glucose degradation products in peritoneal dialysis fluids: do they harm? Kidney Int. 2003; 63(suppl 84): s148-s151.

11. Liu YH, Liu FY, Zhang H, Peng YM, Yuan F, Liu $H$, Chen MC, Zhuo L. Effects of high glucose on the cell proliferation, damage and cytokine in human peritoneal mesothelial cells. Zhong Nan Da Xue Xue Bao Yi Xue Ban. 2006. 31(4):575-579.

12. Hendriks PM, Ho-dac-Pannekeet MM, van Gulik $\mathrm{TM}$ et al. Peritoneal sclerosis in chronic peritoneal dialysis patient: Analysis of clinical presentation, risk factor, and transport kinetics. Perit Dial Int. 1997; 17:136-143.

13. Struijk DG, Krediet RT. European best practice guidelines: adequacy in peritoneal dialysis. Contrib Nephrol 140:170-175, 2003.

14. Códigos EDTA 1994-1995 y su agrupación, extraídos del Informe 2006. Módulo Básico. Subsistema de Insuficiencia Renal Crónica. Servicio Andaluz de Salud. Junta de Andalucía.

15. Twardowski ZJ, Nolph KD, Khanna R, et al. Peritoneal equilibration test. Perit Dial Bull. 1978; 7:138-147.

16. Sociedad Española para el estudio de la Obesidad (SEED0). Consenso SEEDO 2000 para la evaluación del sobrepeso y la obesidad y el establecimiento de criterios de intervención terapéutica. Med Clin (Barc) 2000; 115:587-97.

17. Diez JJ, Iglesias $P$, Selgas R. Diabetes, hiperlipemia y obesidad en una unidad de diálisis peritoneal: Estudio descriptivo transversal. Nefrología. 1995; 15(2):156-62. 